Rev. Élev. Méd. vét. Pays trop., 1977, 30 (4) : 369-372.

\title{
Action trypanocide du chlorhydrate de chlorure d'Isométamidium sur Trypanosoma evansi et essai de traitement de la trypanosomiase du dromadaire
}

\author{
par J. BALIS et D. RICHARD $(* *)$
}

\begin{abstract}
RÉSUMÉ
Le chlorhydrate de chlorure d'Isométamidium est doué de peu d'activité à l'égard de Trypanosoma evansi. En l'absence de produits plus actifs, son uti. lisation chez le dromadaire par injection intraveineuse ou intramusculaire à des doses comprises entre $0,50 \mathrm{et} 1 \mathrm{mg} / \mathrm{kg}$, permet d'attendre durant deux à trois semaines une thérapeutique réellement curative.
\end{abstract}

Le chlorhydrate de chlorure d'Isométamidium est bien connu pour ses excellentes propriétés curatives et préventives à l'égard de la maladie causée par Trypanosoma congolense et Trypanosoma vivax $(4,3)$.

A des doses comprises entre 0,25 et $1 \mathrm{mg} / \mathrm{kg}$ en solution à 1 ou 2 p. 100 , ce trypanocide, injecté par voie intramusculaire profonde, est très bien supporté par les bovins.

Cependant, Trypanosoma brucei et spécialement Trypanosoma evansi sont beaucoup moins sensibles à son action (3).

Le traitement du Surra à l'aide de ce corps, n'a fait l'objet que de quelques publications très différentes quant à leurs conclusions. C'est ainsi que RAGHAVAN et NISRA AHMED KHAN (6) n'observant pas de rechutes après trois semaines d'observation, déclarent avoir obtenu la guérison de bovins par une seule

(*) TRYPAMIDIUM (SPECIA), SAMORIN (May et Baker).

(**) I. E. M. V. T. 10, rue Pierre-Curie, 94700 Maisons-Alfort, France. Mission Vétérinalre Française, Po Box 1053, Addis-Abeba, Ethiopie.

Veterinary Institute, Po Box 19, Debre-Zeit, Ethiopie. injection à la dose de $0,25 \mathrm{mg} / \mathrm{kg}$ en solution à 1 p. 100.

Par contre, SRIVASTAVA et MALHOTRA (8), expérimentant sur des chiens trouvèrent qu'il fallait atteindre une posologie de 0,50 à $1 \mathrm{mg} / \mathrm{kg}$.

Pour CHAND et SINGH (2), des ânes trypanosomés ne réagissent favorablement qu'à des doses comprises entre 1 et $2 \mathrm{mg} / \mathrm{kg}$.

Récemment PETROVSKIJ (5), effectuant en Union Soviétique des essais sur 10 chameaux trypanosomés, déclarèrent, après deux mois d'observation, avoir obtenu leur guérison par un seul traitement à la dose de $1 \mathrm{mg} / \mathrm{kg}$.

En Ethiopie, spécialement dans le sud du pays, le cheptel camelin est très important. On y utilise presque exclusivement la Suramine sodique curativement et préventivement. Cependant une chimiorésistance serait apparue, aussi l'emploi du Sulfate d'Antrycide a-t-il été préconisé.

L'objet du présent travail est d'évaluer l'activité du chlorhydrate de chlorure d'Isométamidium sur Trypanosoma evansi et de définir la posologie à adopter dans le traitement de la maladie chez le dromadaire. 
I. Etude préliminaire de l'action du chlorhydrate de chlorure d'Isométamidium sur Trypanosoma evansi inoculé à des cobayes

Un premier essai a été fait sur 8 animaux fortement parasités. Ils ont été traités par injection sous-cutanée d'une solution à 1 p. 1000 de trypanocide, de façon à éviter d'avoir à injecter des volumes trop faibles.

Les doses suivantes furent administrées sur deux cobayes chaque fois, à l'aide d'une seringue graduée au centième de millilitre.

$$
\begin{array}{lll}
0,25 & \mathrm{mg} / \mathrm{kg} & (0,19 \mathrm{ml}) \\
0,50 & \mathrm{mg} / \mathrm{kg}(0,38 \mathrm{ml}) \\
1 & \mathrm{mg} / \mathrm{kg}(0,75 \mathrm{ml}) \\
2 & \mathrm{mg} / \mathrm{kg}(1,50 \mathrm{ml}) .
\end{array}
$$

Sur chaque animal a été quotidiennement fait un examen à l'état frais complété, en cas de présence des parasites, par des frottis fixés à l'alcool méthylique et colorés au Giemsa. Ces derniers permirent une évaluation de la parasitémie, en faisant au grossissement 600, la moyenne du nombre de trypanosomes observés dans $10,20,50$ ou 100 champs microscopiques selon la fréquence des parasites.

Si cette méthode ne fournit qu'une valeur approximativement proportionnelle au nombre réel, elle permet cependant de suivre correctement l'évolution de la parasitémie et présente, outre sa rapidité, l'avantage de pouvoir être exécutée ultérieurement.

Les résultats de cette expérience confirment le fait que l'activité du chlorhydrate de chlorure d'Isométamidium sur Trypanosoma evansi est environ quatre à cinq fois plus faible que celle observée chez le zébu par d'autres auteurs (4) sur les flagellés des groupes vivax et congolense.

En effet, sur deux cobayes présentant une parasitémie de 9 à 10 trypanosomes par champ et traités à la dose de $0,25 \mathrm{mg} / \mathrm{kg}$, des frottis effectués plusieurs fois par jour firent apparaître un début d'activité trypanocide vers la dixième heure mais il n'y eut pas disparition des parasites et deux jours après le traitement, on en observait encore un pour quatre champs. Par la suite, leur nombre augmenta régulièrement pour atteindre, après une dizaine de jours, la valeur de 7 à 8 .

Les doses de 0,50 et $1 \mathrm{mg} / \mathrm{kg}$ ne semblèrent pas beaucoup plus actives puisqu'elles n'abou- tirent qu'à une diminution très sensible de la parasitémie avec parfois absence momentanée de flagellés.

Seule une posologie de $2 \mathrm{mg} / \mathrm{kg}$ fut plus efficace puisqu'après 24 heures la disparition des trypanosomes était totale. Cette dernière persista sur un animal pendant deux mois que dura l'observation mais, pendant la même période, l'autre cobaye présenta une fois quelques rares parasites.

Ce travail a été repris avec comme objectif de provoquer l'apparition d'une résistance par un traitement à la dose de $0,25 \mathrm{mg} / \mathrm{kg}$ de toutes les rechutes.

Six cobayes fortement parasités furent soumis aux posologies suivantes:

$$
\begin{array}{llll}
3 & \text { à } & 0,25 & \mathrm{mg} / \mathrm{kg} \\
1 \text { à } & 0,50 & \mathrm{mg} / \mathrm{kg} \\
1 \text { à } & 1 & \mathrm{mg} / \mathrm{kg} \\
1 \text { à } & 2 & \mathrm{mg} / \mathrm{kg} .
\end{array}
$$

Parmi les 3 animaux ayant reçu $0,25 \mathrm{mg} / \mathrm{kg}$, l'un en observation durant 3 mois présenta de multiples rechutes de plus en plus rapprochées, $c^{\prime}$ 'est-à-dire aux $10^{\mathrm{e}}, 9^{\mathrm{e}}, 5^{\mathrm{e}}, 3^{\mathrm{e}}, 4^{\mathrm{e}}, 2^{\circ}$ et $2^{\mathrm{e}}$ jours après traitement. Lors de chacune d'elle, le traitement n'a été appliqué que lorsque la parasitémie était redevenue importante, c'està-dire respectivement $9-6-6-5-5-12-6-$ trypanosomes par champ. Vers la fin de l'expérience, l'Isométamidium semblait ne plus être efficace.

Les 2 autres animaux traités à $0,25 \mathrm{mg} / \mathrm{kg}$ ont présenté une trypanosomiase différente : l'un a fait une rechute après 18 jours ; un traitement fit disparaître les parasites pendant 17 jours puis une forte poussée (15 par champ) fut aisément curable. L'autre a fait une première rechute à 22 jours (12 parasites par champ) et un traitement fut complètement efficace.

Aux autres posologies, les résultats suivants furent observés :

— à $0,50 \mathrm{mg} / \mathrm{kg}$ : rechute à 48 jours ; après traitement, nouvelle rechute au $19^{\circ}$ jour ;

- à $1 \mathrm{mg} / \mathrm{kg}$ : rechute au $50^{\circ}$ jour; à la suite du traitement, nouvelle rechute 16 jours après ;

- à $2 \mathrm{mg} / \mathrm{kg}$ : pas de rechute et après trois mois d'observation, l'état général de l'animal était excellent. 
En conclusion, il semble possible qu'une répétition de faibles doses soit capable d'induire assez rapidement une résistance au trypanocide.

\section{Expérimentation sur le dromadaire}

Elle a été conduite d'une part à Dire-Dawa dans la province du Harar avec la collaboration des Docteurs-vétérinaires DESROTOUR, BLANC, BOUVIER, DESLANDES, GERBALDI et LIDOVE, d'autre part à Neghele dans la sous-province du Borana avec l'aide du Docteur-vétérinaire GUIDOT, de l'assistant GUEBRE-NEGUS et du vaccinateur ISAHYES DAGEIRO.

Les essais d'efficacité effectués sur cobaye nous ont amené à penser que si l'on veut, au minimum, obtenir un effet curatif chez le dromadaire, les doses employées doivent être de l'ordre de $1 \mathrm{mg} / \mathrm{kg}$. Nous avons cependant choisi l'éventail suivant : $0,50-1-2 \mathrm{mg} / \mathrm{kg}$ et considéré que la voie intraveineuse devait permettre de réduire la quantité de trypanocide à injecter.

A) Toxicité du chlorhydrate de chlorure d'Isométamidium chez le dromadaire

La posologie adoptée étant relativement élevée, il était nécessaire de contrôler la toxicité de l'Isométamidium en fonction de la voie d'administration et de la dose employée :

Voie sous-cutanée :

0,5 et $1 \mathrm{mg} / \mathrm{kg}$ : légère fluctuation douloureuse pendant 3 à 4 jours.

$2 \mathrm{mg} / \mathrm{kg}$ : fluctuation assez importante douloureuse pendant une semaine.

Voie intramusculaire :

$0,5 \mathrm{mg} / \mathrm{kg}$ : l'injection est correctement tolérée bien que douloureuse pendant plusieurs jours.

$1 \mathrm{mg} / \mathrm{kg}$ : l'injection provoque une douleur plus importante aboutissant à une boiterie si l'on opère au niveau de la fesse ou une gêne de l'encolure si la piqûre est faite à ce niveau.

$2 \mathrm{mg} / \mathrm{kg}$ : mêmes conséquences que précédemment mais douleur encore plus importante.

\section{Voie intraveineuse:}

Elle est anodine aux doses de 0,5 et $1 \mathrm{mg} / \mathrm{kg}$ à la condition expresse que l'injection soit faite lentement et en évitant le mélange sang-trypanocide dans la seringue (1).
A la dose de $2 \mathrm{mg} / \mathrm{kg}$ et bien que les mêmes précautions soient prises, on observe un choc assez important avec chute de l'animal, ptyalisme, larmoiement, diarrhée et forte accélération du rythme cardiaque.

B) Activité du chlorhydrate de chlorure d'Isométamidium sur la trypanosomiase à Trypanosoma evansi du dromadaire

Cette activité a été recherchée spécialement après injection intraveineuse du trypanocide et surtout à la dose de $1 \mathrm{mg} / \mathrm{kg}$ qui s'avère être la limite de sécurité et de rentabilité par rapport aux autres trypanocides. Deux dromadaires ont cependant été traités par injection intramusculaire.

Nous avons utilisé des animaux d'un poids compris entre 200 et $300 \mathrm{~kg}$ dont 3 furent splénectomisés (7). Tous furent inoculés avec une souche de Trypanosoma evansi entretenue sur cobayes. Cette souche était très pathogène puisqu'elle tua 3 dromadaires splénectomisés 4,5 et 9 jours après l'inoculation du parasite.

\section{Résultats :}

Par voie intraveineuse :

$0,5 \mathrm{mg} / \mathrm{kg}$ : disparition des trypanosomes en 3 jours puis réapparition 15 jours après le traitement. $36 \mathrm{~h}$.

$1 \mathrm{mg} / \mathrm{kg}$ : disparition des trypanosomes en

Premier animal : rechute au bout de 60 jours.

Deuxième animal : (splénectomisé) : réapparition fugace après 19 jours puis rechute grave au $71^{e}$ jour.

Troisième animal : rechute au bout de 71 jours.

Quatrième animal : toujours négatif après 169 jours.

$2 \mathrm{mg} / \mathrm{kg}$ : disparition des trypanosomes en $24 \mathrm{~h}$, toujours négatif après 105 jours.

\section{Par voie intramusculaire :}

Les résultats obtenus sont comparables aux précédents : l'injection d'Isométamidium a fait disparaître les parasites en $24 \mathrm{~h}$ mais les deux animaux ont rechuté, l'un 32 jours, l'autre 53 jours après le traitement. 


\section{CONCLUSION}

De cette expérimentation conduite à la fois" sur cobayes et dromadaires, nous pouvons conclure que le chlorhydrate de chlorure d'Isométamidium n'est que médiocrement actif sur Trypanosoma evansi. Il faut, en effet, en arriver à une dose de $2 \mathrm{mg} / \mathrm{kg}$ pour obtenir la guérison.

La voie intraveineuse, utilisable sans danger jusqu'à $1 \mathrm{mg} / \mathrm{kg}$, à condition d'injecter le trypanocide lentement et d'une façon continue, provoque des chocs impressionnants dès que l'on atteint une posologie de $2 \mathrm{mg} / \mathrm{kg}$. Ces chocs ne surviennent pas si l'Isométamidium est administré par voie intramusculaire, même à des doses supérieures à $2 \mathrm{mg} / \mathrm{kg}$, mais c'est alors qu'une douleur importante apparaît et de surcrốt, s'il s'agit d'un animal de boucherie, l'Isométamidium, de par son amertume, rend impropre à la consommation une partie de la viande.

Enfin des traitements répétés semblent susceptibles d'induire assez rapidement une résistance.

Le chlorhydrate de chlorure d'Isométamidium ne doit donc être utilisé qu'en cas d'urgence afin d'arrêter momentanément l'évolution de la maladie si l'on ne dispose pas d'un trypanocide plus actif.

\section{SUMMARY}

Trypanocide action of Isometamidium chloride hydrochlorate on Trypanosoma evansi and an attempt to control trypanosomiasis in dromedary

The efficiency of Isometamidium chloride hydrochlorate in controlling Trypanosoma evansi is reduced. But in the absence of other avalable efficient product when the dromedary is treated with intravenous and intramuscular injections at rates varying from 0.50 to $1 \mathrm{mg} / \mathrm{kg}$, it is possible to wait two or three weeks for a truly curative treatment.

\section{RESUMEN}

\section{Acción tripanocida del cloridrato de cloruro de Isometamidium sobre Trypanosoma evansi y ensayo de tratamiento de la tripanosomiasis del dromedario}

La actividad del cloridrato de cloruro de Isometamidium contra Trypanosoma evansi es reducida.

En el dromedario, la inyección intravenosa o intramuscular de dosis de 0,50 a $1 \mathrm{mg} / \mathrm{kg}$ permite esperar durante dos o tres semanas una terapéutica efectivamente curativa.

\section{BIBLIOGRAPHIE}

1. BALIS (J.). Note sur la toxicité de l'Isométamidium par injection intraveineuse chez quelques mammifères domestiques et spécialement chez le dromadaire. Rev. Elev. Méd, vét. Pays trop., 1977, 30 (4) : 363-365.

2. CHAND (K.), SINGH (R. P.). Therapeutic effect of Samorin in donkeys and dogs experimentally infected with Trypanosoma evansi. Indian vet. J., 1970, 47 : 475-479.

3. FINELLE (P.). Chimiothérapie et chimioprévention de la trypanosomiase animale. Acqussitions récentes et situation actuelle. Cah. Méd. vét., 1973, 42 (5) : 215-226.

4. FINELLE (P.), LACOTTE (R.). Action trypanocide de deux sels d'Isométamidium. Rev. Elev, Méd. vét. Pays trop., 1963, 16 (4) : 405.

5. PETROVSKIJ (V. V.). Problèmes actuels de l'éradication des trypanosomes. Veterinariya, 1974, 5: 68-70.

6. RAGHAVAN (R. S.), KHAN (N. A.). Bovine trypanosomiasis. Control with Samorin. Indian vet. J., 1970, 47: 187-188.

7. RICHARD (D.). Une méthode de splénectomie chez le dromadaire. (A paraittre.)

8. SRIVASTAVA (R.V. N.), MALHOTRA (N. N.). Efficacy of Mel W-Trimelarsan-Melarsonyl Potassium and Samorin-Isometamidium chloride against Trypanosoma evansi experimental infection in dogs. Indian J. anim. Hlih, 1967 : 291-297. 